\title{
Intrinsic Explanations and Numerical Representations
}

\author{
M. Eddon
}

\section{Introduction}

In his (1980), Hartry Field argues that good explanations of physical phenomena are "intrinsic explanations." Roughly, an intrinsic explanation of some phenomenon is one that invokes objects that are causally relevant to the phenomenon to be explained. For instance, an explanation of the structure of spacetime that appeals to spacetime points and the relations they stand in is an intrinsic explanation, while one that appeals to causally irrelevant entities like numbers is an extrinsic explanation. More carefully, let us say that a predicate $F$ is an intrinsic predicate iff whether $F\left(x_{1}, \ldots, x_{n}\right)$ obtains does not depend on anything other than $x_{1}, \ldots, x_{n}$, and the relations among them. Let us say that a fact is an intrinsic fact iff the predicates it involves are intrinsic predicates. Finally, let us say that an explanation is an intrinsic explanation iff it only involves intrinsic facts and intrinsic predicates. ${ }^{1}$

Field argues that his treatment of quantity is able to provide intrinsic explanations of the structure of space, spacetime, and other quantitative properties, as well as intrinsic explanations of why certain numerical representations of quantities (distances, lengths, mass, temperature, etc.) are appropriate or acceptable while others are not.

In contrast, Brian Ellis (1960) and (1966) argues that certain quantitative predicates are not intrinsic, ${ }^{2}$ and that numerical representations of quantitative features are largely a matter of convention. In a similar vein, Peter Milne (1986) uses arguments like Ellis's to argue that both of Field's claims are false - that Field's account cannot provide intrinsic explanations of either our numerical representations of quantity or the structure of quantity.

In this paper, I show where the arguments put forth by Ellis and Milne go wrong, and where they go right. Their arguments that one cannot provide an

\footnotetext{
${ }^{1}$ These notions of "intrinsic" are those employed by Field and Milne. They are not particularly explicit about what they mean by these terms, but the characterizations given above are suggested by the passages in Field (1980, 27-28 and 41-46) and Milne (1986, 344 and 346). (Of course, to make these characterizations completely precise, one needs to spell out the relevant notions of "involves" and "depends.")

${ }^{2}$ Ellis does not put it in quite this way. Rather, he suggests that "grounds of convenience" (1966, 82 ) and "the roles of the various quantities in physical theory" (86) are "the only kind[s] of justification that can be given" (83) for the choice of fundamental scale and fundamental measuring procedure. This entails that certain length relations are, on his view, not intrinsic.
} 
account of quantity in "purely intrinsic" terms fail for the same reason: they take the conventionality of numerical representations of quantitative features to reveal conventionality of the features themselves. Both Ellis and Milne infer from the fact that choice of measurement scale is a matter of convention that the underlying quantitative features the scale represents must also be a matter of convention.

On the other hand, Ellis's claim that the numerical representations of quantitative features that we employ are merely conventional, and Milne's claim that Field's framework does not provide an intrinsic explanation of our use of certain numerical representations over others, are both right. I will show that Field is mistaken to claim this as a virtue of his framework. That said, I will tentatively suggest a way to modify Field's framework so that it can provide such intrinsic explanations.

In sections (2) and (3), I present two questions Ellis raises about measurement, concerning the structure of quantity and the numerical representation of quantity. In section (4), I show how, contrary to what Ellis claims, these questions are orthogonal to one another, and I show how this undercuts Ellis's argument that one cannot provide an account of quantity in "purely intrinsic" terms. In section (5), I describe Field's framework. In sections (6) and (7), I present and assess Milne's objections to it. Finally, in section (8), I suggest one way to modify Field's framework so that it can provide an intrinsic explanation for why some numerical representations are better than others.

\section{Two Fundamental Measuring Procedures}

Consider three rods $-a, b$, and $c$. Suppose that $a$ and $b$ are the same length. When placed end to end, the distance from the leftmost tip of $a$ to the rightmost tip of $b$ is the same as the length of $c$ :

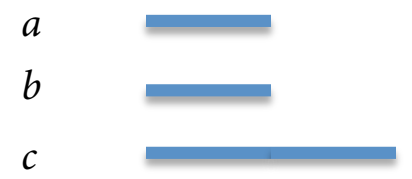

This way of measuring the lengths of rods is what Brian Ellis calls a "measuring procedure." ${ }^{3}$ Roughly, a measuring procedure is a physical procedure for adding objects together, with respect to some magnitude. ${ }^{4}$ Let us call the particular

\footnotetext{
${ }^{3}$ See Ellis (1960) and (1966, 74-89).

${ }^{4}$ Ellis writes: "All fundamental measurement depends upon the existence of a procedure for producing equality in respect of the fundamentally measureable quantity. In the fundamental measurement of length, the object to be measured is equaled in length by a line produced by a
} 
measuring procedure used above the "standard measuring procedure." When $a$ is added to, or concatenated with, $b$ using the standard measuring procedure, the result is $c$. And it seems plainly clear that when two rods of equal length are added together, the resulting rod is twice as long as each of the individual rods. So, it seems plainly clear that we can deduce from this procedure that $c$ is twice as long as $a$ and $b$.

But is that so? Consider a different measuring procedure. Begin again with rods $a$ and $b$, and now add another rod, $d$. Suppose that instead of placing $a$ and $b$ end-to-end, we place them perpendicular to one another. When perpendicular to one another, the distance from the leftmost tip of $a$ to the topmost tip of $b$ is the length of $d$ :

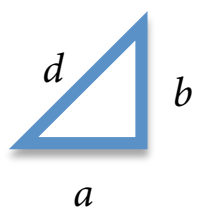

Call this way of adding or concatenating objects the "right-angle measuring procedure." When $a$ is added to, or concatenated with, $b$ using the right-angle measuring procedure, the result is $d$. And if we assume, as we did above, that concatenating two objects of equal length yields an object that is twice as long, then it seems we are committed to saying that $d-\operatorname{not} c$ - is twice as long as $a$ and b. ${ }^{5}$

So is the length of $a$ and $b$ added together the same as the length of $c$, or is it the same as the length of $d$ ? Is there some reason to think that the standard measuring procedure, and not the right-angle measuring procedure, is the "correct" measuring procedure? This is one of two questions Ellis poses in his (1960) and (1966, 74-86):

series of repeated procedures with a standard or set of standard objects (i.e. measuring rods)." (Ellis 1960, 42)

${ }^{5}$ Suppose one claims that only $c$ is twice as long as $a$ and $b$, and this is why the standard measuring procedure is the correct one to use. But whether $c$ really is twice the length of $a$ and $b$, irrespective of some choice of measuring procedure, is precisely the question at issue. As Ellis notes, to maintain that "the notion of 'twice as long as' has a significance which is independent of measuring operations" (Ellis 1966, 83) is simply to say that there is an objectively correct measuring procedure. If the standard measuring procedure is the fundamental one, then $c$ is twice as long as $a$ and $b$; while if the right-angle measuring procedure is the fundamental one, then $d$ is twice as long as $a$ and $b$. And if there is no fact of the matter about which of these is fundamental, then there is no fact of the matter as to which rod is twice as long as $a$ and $b$. 
Question 1: What is the fundamental measuring procedure? Is there some reason to think that the fundamental measuring procedure is the standard one as opposed to the right-angle one?

What does Ellis mean by a "fundamental measuring procedure"? A measuring procedure is a physical procedure for adding together the lengths of objects. Of course, there are many different procedures one may carry out to measure length. What makes a particular measuring procedure fundamental is that it tracks the fundamental facts about length. In other words, the fundamental measuring procedure is interesting because its method of "adding" rods lines up with the fundamental concatenation relation. ${ }^{6}$ So, Ellis's question about which fundamental measuring procedure is the correct one to use is, at bottom, a question about the fundamental concatenation relation. Here is Ellis's first question, reformulated:

Question 1, reformulated: What is the fundamental concatenation relation? Is there some reason to think that it is the one that lines up with the standard measuring procedure, where rods are placed end-to-end, as opposed to the one that lines up with the right-angle measuring procedure, where rods are placed perpendicular to one another?

In what follows, I use "measuring procedure" and "concatenation relation" (or "concatenation predicate") interchangeably.

\section{Scales of Measurement}

How might one argue that the standard concatenation relation (or, in Ellis's terms, the "standard measuring procedure") is the one linked to the objective notion of length, while the right-angle concatenation relation (or, in Ellis's terms, the "right-angle measuring procedure") is not? One thought is to appeal to our use of certain scales of measurement. Consider, for instance, the inches scale. Suppose that $a$ and $b$ are both one inch long, and $c$ is two inches long. So $a$ and $b$ are assigned the number 1 on the inches scale, and $c$ is assigned the number 2 . Now, one might say, the inches scale accords with the standard concatenation relation in an important way. The concatenation of $a$ and $b$, each one inch long, yields an object that is two inches long. More generally, the inches scale is additive over the standard concatenation relation: when the lengths of rods are

\footnotetext{
${ }^{6}$ In this context, I use "fundamental" to mean something akin to Lewis's "perfectly natural." See Lewis (1983).
} 
measured in inches, the number assigned to the concatenation of $x$ and $y$ is the sum of the numbers assigned to $x$ and $y$ individually.

In contrast, the inches scale is not additive over the right-angle concatenation relation. The right-angle concatenation of $a$ and $b$ is $d$, which is $\sqrt{2}$ inches long. So the number assigned to $d$ is not the sum of the numbers assigned to $a$ and $b(1+1 \neq \sqrt{2})$. Therefore, one might claim, since we want our scale to be additive over the fundamental concatenation relation, the right-angle concatenation relation is to be rejected. ${ }^{7}$

As Ellis points out, however, this attempt to justify the standard concatenation relation is unsuccessful. There are two reasons. First, although it is true that the inches scale is not additive over the right-angle concatenation relation, one can construct scales that are additive. Ellis offers one candidate: the so-called dinches (or "diagonal inches") scale. The dinches scale is related to the inches scale in the following way:

$$
\begin{aligned}
& 1 \text { inch }=1 \text { dinch } \\
& 2 \text { inches }=4 \text { dinches } \\
& 3 \text { inches }=9 \text { dinches }
\end{aligned}
$$

and more generally:

$$
n \text { inches }=n^{2} \text { dinches }
$$

Since $a$ and $b$ are both one inch long, they are also both one dinch long, and so are assigned the number 1 on the dinches scale. And $d$ - the hypotenuse of the right triangle formed by concatenating $a$ and $b$ using the right-angle measuring procedure - is two dinches long, and so is assigned the number 2 on the dinches scale. The number assigned to $d$ is the sum of the numbers assigned to $a$ and $b$ $(1+1=2)$, and so we see that the dinches scale is additive over the right-angle concatenation relation.

So, even if we grant the assumption that a satisfactory measurement scale must be additive over the fundamental concatenation relation, this does not give us a reason to reject the right-angle concatenation relation. For we can construct a scale - the dinches scale - that is additive over the right-angle concatenation relation. Regardless of which concatenation relation is the fundamental one, we can construct a scale that is additive over that relation.

The second reason this attempt to defend the standard concatenation relation falters is that the assumption that an adequate measurement scale be additive over the fundamental concatenation relation is unjustified. Why must our scale be additive in this way? There are plenty of satisfactory scales that seem

\footnotetext{
${ }^{7}$ This appears to be the move suggested by Fox (2007).
} 
to fail to satisfy this criterion: decibels (of sound), Richter magnitudes (for earthquakes). As Ellis $(1960,47)$ points out, the requirement that certain measurement scales be additive seems to be a matter of convention more than anything else. ${ }^{8}$

That said, the dinches scale surely seems odd, and it would be interesting if there were some independent justification for rejecting it. This leads to the second question Ellis poses:

Question 2: $\quad$ What is the correct measurement scale to use when measuring lengths? Is there some reason to think that scales like the inches scale are the correct ones to use as opposed to scales like the dinches scale?

\section{Two Paths to a Dinches Scale}

Ellis takes these two questions - the question of the correct fundamental measuring procedure, and the question of the correct measurement scale - to be related to one another, such that an answer to one of these questions entails an answer to the other. ${ }^{9}$ In particular, Ellis says that the intrinsic facts about lengths do not give us any reason to use one measurement scale over another, and therefore that the intrinsic facts about lengths do not give us any reason to use one concatenation relation over another. But in fact these two questions are orthogonal to one another. In this section, I show how one can construct both inches and dinches scales from the standard concatenation relation, and also how one can construct both inches and dinches scales from a non-standard concatenation relation. In sections (6) and (7), this result is used to evaluate Milne's objections to Field.

Let us begin with the canonical treatment of measurement. Following Krantz et al. (1971), there are two primitive predicates: greater than or equal to $(\succeq)$ and concatenation (o). To say that $x \succeq y$ means, intuitively, that the length of $x$ is greater than or equal to the length of $y$. And to say that $\circ(x, y, z)$ means, intuitively, that the length of $z$ is the length of $x$ concatenated with the length of $y$. The concatenation predicate, $\circ$, corresponds to what Ellis calls the standard

\footnotetext{
${ }^{8}$ See also Krantz et al. $(1971,100)$.

${ }^{9}$ Ellis: "The two problems of choice involved in fundamental measurement are, of course, very closely linked together. For to choose a fundamental scale for the measurement of any quantity, both of these choices have to be made. Consequently, they are both parts of the more general problem of choosing a scale, and the same considerations are likely to be relevant to both choices." (Ellis 1966, 81) See also remarks in Ellis (1960, 46-47) and 1966 (82-86).
} 
fundamental measuring procedure.

Consider a set of rods, $A$, over which the length relations $\succeq$ and $\circ$ are defined. In order to represent the objects in $A$ using numbers, we want a function that maps the relational structure $\langle A, \succeq, 0\rangle$ to some numerical structure. A natural way to do this is to carry the objects in $A$ to the positive real numbers, carry $\succeq$ to $\geq$, and carry $\circ$ to + . One can then prove representation and uniqueness theorems - theorems specifying the constraints on numerical representations of the objects and relations of $A$. In this case, the representation theorem states that $\langle A, \succeq, 0\rangle$ is a model of the axioms of extensive measurement iff there is a function $\phi_{1}$ from $\left\langle A, \succeq, 0>\right.$ into $<\mathrm{Re}^{+}, \geq$, $+>$such that:

(a $) x \succeq y$ iff $\phi_{1}(x) \geq \phi_{1}(y)$

$\left(b_{1}\right) \circ(x, y, z)$ iff $\phi_{1}(x)+\phi_{1}(y)=\phi_{1}(z)$

The corresponding uniqueness theorem states that for any two functions $\phi_{1}$ and $\phi_{1}{ }^{\prime}$ defined over $A, \phi_{1}$ and $\phi_{1}{ }^{\prime}$ satisfy these constraints iff they differ only by a positive multiplicative constant (i.e., $\phi_{1}=n \phi_{1}{ }^{\prime}$ where $n>0$ ). This uniqueness theorem captures the fact that if the inches scale, say, is an acceptable measurement scale for lengths, then so is the centimeters scale, the yards scale, the miles scale, and so on. (Of course, some of these scales may be more convenient in some circumstances. If you ask someone how far away the moon is from the earth, you might be irritated if she gives you the answer in millimeters.)

While this may be the most obvious way to numerically represent the objects in $A$, it is not the only one. We could, for instance, choose to map $<A$, $\succeq$, o> onto $<\mathrm{Re}^{+}, \geq$, \#>, where \# is defined as follows:

$n \# o=p$ iff $(\sqrt{ } n+\sqrt{ } o)^{2}=p$ (where $n, o$, and $p$ are variables ranging over numbers)

In this case, one can prove a representation theorem that states that $\langle A, \succeq$, 0 is a model of the axioms iff there is a function $\phi_{2}$ from $\langle A, \succeq$, 0$\rangle$ into $<\mathrm{Re}^{+}, \geq$, \# that satisfies the following analogues of $\left(a_{1}\right)$ and $\left(b_{1}\right)$ above:

$\left(a_{2}\right) x \succeq y$ iff $\phi_{2}(x) \geq \phi_{2}(y)$

$\left(b_{2}\right) \circ(x, y, z)$ iff $\phi_{2}(x) \# \phi_{2}(y)=\phi_{2}(z)$

The corresponding uniqueness theorem is the same as the one above: for any two functions $\phi_{2}$ and $\phi_{2}{ }^{\prime}$ defined over $A, \phi_{2}$ and $\phi_{2}{ }^{\prime}$ satisfy these constraints iff they differ only by a positive multiplicative constant (i.e., $\phi_{2}=n \phi_{2}{ }^{\prime}$ where $n>0$ ).

$\phi_{1}$ and $\phi_{2}$ are functions that map the same relational structure, $\langle A, \succeq, \circ\rangle$, onto different numerical structures. And they are related in the following way: $\phi_{1}$ $=\phi_{2}^{2}$. If $\phi_{1}$ is the inches scale, then $\phi_{2}$ is the dinches scale. So we see that the relational structure in which the standard concatenation predicate figures permits the construction of both inches and dinches scales. 
Now let's see how one can construct both inches and dinches scales from a non-standard concatenation relation. Suppose that instead of $\circ$, we introduce a different sort of concatenation predicate, ${ }^{\star}$. Intuitively, ${ }^{\star}$ corresponds to Ellis's right-angle measuring procedure. Consider again our set of rods, $A$, but rather than define $\succeq$ and $\circ$ over $A$, we define $\succeq$ and ${ }^{\star}$ over $A$. As before, in order to represent the objects in $A$ using numbers, we want a function that maps the relational structure $\left\langle A, \succeq,{ }^{*}\right\rangle$ to some numerical structure. One way to do this is to carry the objects in $A$ to the positive real numbers, carry $\succeq$ to $\geq$, and carry ${ }^{\star}$ to + . The representation theorem in this case states that $\left\langle A, \succeq,{ }^{*}\right\rangle$ is a model of the axioms iff there is a function $\varphi_{1}$ from $\left.<A, \succeq,{ }^{*}\right\rangle$ into $<\operatorname{Re}^{+}, \geq,+>$such that:

$\left(a_{1}\right) x \succeq y$ iff $\varphi_{1}(x) \geq \varphi_{1}(y)$

$\left(b_{1}\right){ }^{\star}(x, y, z)$ iff $\varphi_{1}(x)+\varphi_{1}(y)=\varphi_{1}(z)$

The corresponding uniqueness theorem states that, for any two functions $\varphi_{1}$ and $\varphi_{1}{ }^{\prime}$ defined over $A, \varphi_{1}$ and $\varphi_{1}{ }^{\prime}$ satisfy these constraints iff they differ only by a positive multiplicative constant (i.e., $\varphi_{1}=n \varphi_{1}^{\prime}$ where $n>0$ ).

Just as $\langle A, \succeq$, $>>$ could be mapped to various numerical structures, so can $\left\langle A, \succeq,{ }^{\star}\right\rangle$. Suppose that instead of carrying ${ }^{\star}$ to + , we carry ${ }^{\star}$ to a different mathematical procedure, $\$$, where $\$$ is defined as follows:

$n \$ o=p$ iff $\sqrt{ }\left(n^{2}+o^{2}\right)=p \quad$ (where $n, o$, and $p$ are variables ranging over numbers)

Now one can prove that $\left\langle A, \succeq,{ }^{*}\right\rangle$ is a model of the axioms iff there is a function $\varphi_{2}$ such that:

$\left(a_{2}\right) x \succeq y$ iff $\varphi_{2}(x) \geq \varphi_{2}(y)$

$\left(b_{2}\right){ }^{\star}(x, y, z)$ iff $\varphi_{2}(x) \$ \varphi_{2}(y)=\varphi_{2}(z)$

$\varphi_{1}$ and $\varphi_{2}$ are functions that map the same relational structure, $\left\langle A, \succeq,{ }^{*}\right\rangle$, onto different numerical structures. And they are related in the following way: $\varphi_{2}=\varphi_{1}^{2}$. If $\varphi_{2}$ is the inches scale, then $\varphi_{1}$ is the dinches scale. So we see that a structure using the right-angle concatenation predicate, ${ }^{*}$, permits the construction of both inches and dinches scales.

Ellis takes the existence of the dinches scale to demonstrate that distances among lengths is not an intrinsic matter - it is not settled by the objects and the length relations that hold among them. If it is settled at all, he believes, it can only be due to considerations of simplicity of mathematical formulations of the laws of nature. ${ }^{10}$ But we see now that this doesn't follow. The fact that we can construct non-standard measurement scales like the dinches scale implies nothing about the underlying length relations, since both dinches and inches scales can be

\footnotetext{
${ }^{10}$ See Ellis $(1966,82-83)$.
} 
constructed from structures with the standard concatenation predicate as well as structures with the right-angle concatenation predicate. True, the inches scale is more convenient and familiar than the dinches scale. But both are just numerical representations - and there are no metaphysical insights to be gleaned from the obvious fact that some numerical representations are more convenient and familiar than others.

\section{Hartry Field and Intrinsic Explanations}

Like Ellis, Milne takes the existence of the dinches scale to show that certain facts about lengths are not "intrinsic facts," or "facts about the world which are statable independently of... the use of particular mathematical representations." (Milne 1986, 341) Milne's criticisms are directed at Field's claim that his framework provides "intrinsic explanations" - explanations that appeal only to "intrinsic facts" - of relevant phenomena.

Field takes his framework to provide intrinsic explanations of two sorts of things: first, an intrinsic explanation of our standard numerical representations of lengths, mass, temperature, and so on; ${ }^{11}$ second, an intrinsic explanation of the structure of space, spacetime, and various scalar magnitudes. ${ }^{12}$

Milne argues that Field's framework fails on both counts. In broad outline, Milne's argument goes as follows: first he argues that Field's framework fails to provide intrinsic explanations of our standard numerical representations

\footnotetext{
11 " $[\mathrm{O}]$ ne of the things that gives plausibility to the idea that extrinsic explanations are unsatisfactory if taken as ultimate explanation is that the functions invoked in many extrinsic explanations are so arbitrary. For example, in the case of geometry, the choice of one distance function over any other one which differs from it by positive multiplicative constant is completely arbitrary; it reflects in effect an arbitrary choice of units for distance." (Field 1980, 45) Concerning his treatment of Euclidean geometry, Field writes that the associated uniqueness theorem "gives an explanation of the fact that the laws of Euclidean geometry, when stated in terms of coordinates, are invariant under shift of origin, reflection, rotation, and multiplication of all distances by a constant factor." (Field 1980, 50)

${ }^{12}$ Concerning his treatment of Newtonian space-time, Field writes: "The position that we arrive at, then, is that the only spatio-temporal relations needed to describe Newtonian space-time are the three invoked in this axiom system... [T] he coordinate system and the distance function can be viewed as merely devices for deriving conclusions about spatio-temporal betweenness, simultaneity, and spatial congruence, conclusions which could be derived without ever bringing in numbers at all." (Field 1980, 53) Concerning temperature, Field says that the relevant question is, "what must the intrinsic facts about temperature differences between physical objects be if it is appropriate to think of temperature as being represented by real numbers?" $(1980,58)$ And after providing his account of these intrinsic facts, he writes: "We have specified the continuity of temperature with respect to space-time in a completely intrinsic way, a way that never mentions spatio-temporal coordinates or temperature scales. In my view this fully intrinsic character of the method makes it very attractive even independently of nominalistic scruples." (Field 1980, 63)
} 
of various quantities. Then he infers that some of the predicates invoked by Field are not intrinsic, and thus any explanations involving them are not intrinsic explanations.

In the following sections, I show that while it's true that Field's framework does not provide an intrinsic explanation of our use of certain numerical representations, this does not entail that it fails to provide intrinsic explanations of the structure of space, spacetime, and various scalar magnitudes. I begin with a rough sketch of Field's framework, and then show where Milne's criticisms of Field go awry.

\subsection{Field's Framework}

Field's treatment of space, spacetime, and other quantitative properties is modeled on Hilbert's axiomatization of Euclidean geometry. Let's here consider Field's treatment of Euclidean geometry (in section (7) we see how this account is modified to apply to other quantitative properties). Field takes the basic predicates to be the 3-place predicate betweenness (Bet) and the 4-place predicate congruence (Cong). To say that $y$ Bet $x z$ means, intuitively, that the spatial location of $y$ is between that of $x$ and $z$. And to say that $x y$ Cong $z w$ means, intuitively, that the spatial distance between $x$ and $y$ is the same as that between $z$ and $w \cdot{ }^{13}$

One can provide representation and uniqueness theorems for Field's account of Euclidean geometry. Roughly, the representation theorem says that, given a domain of points $A$ over which betweenness and congruence are defined, there is a function $d$ that maps pairs of points onto numbers such that:

(a) for any points $x, y, z$, and $w, x y$ Cong $z w$ iff $d(x, y)=d(z, w)$

(b) for any points $x, y$, and $z, y$ Bet $x z$ iff $d(x, y)+d(y, z)=d(x, z)$

The corresponding uniqueness theorem for betweenness and congruence says that, given a model of the axiom system and any two functions $d$ and $d^{\prime}$ defined over $A$, if $d$ satisfies $(a)$ and $(b)$, then $d^{\prime}$ satisfies $(a)$ and $(b)$ iff $d$ and $d^{\prime}$ differ by a positive multiplicative constant.

Field takes these representation and uniqueness theorems to have philosophical significance. The representation theorem, he says, "shows that statements that talk about space alone, without reference to numbers, are equivalent to certain 'abstract counterparts' which do talk about numbers." (Field $1980,27)$ So, if we think of $d$ as a distance function (as intuitively it is), then we see that all the geometrical laws stated in terms of numbers can be restated in

\footnotetext{
${ }^{13}$ Field (1980, 25-26)
} 
terms of betweenness and congruence. The uniqueness theorem guarantees that the inches scale satisfies $(a)$ and $(b)$ iff the centimeters scale does as well, since they differ by only a multiplicative constant. Field takes this to show that, given his treatment of geometry, "the fact that geometric laws, when formulated in terms of distance, are invariant under multiplication of all distances by a positive constant, but are not invariant under any other transformation of scale, receives a satisfying explanation: it is explained by the intrinsic facts about physical space, i.e. by the facts about physical space which are laid down without reference to numbers in Hilbert's axioms." (Field 1980, 27) ${ }^{14}$

So, given Field's account, we can prove that there is a range of numerical representations that satisfy certain constraints. This, says Field, shows that we are justified in using the numerical representations that we do. In other words, he claims that his account provides an intrinsic explanation for why certain numerical representations of distance, length, etc., are permissible while others are not.

\section{Milne's First Objection: Differential Congruence}

In section (4), we saw that different kinds of numerical representations of length can be constructed independently of the underlying length relations. So we already have reason to be skeptical of Field's claim that the length facts themselves explain why we use one representation rather than another. And indeed, Milne challenges Field's claim, showing that one can numerically represent betweenness and congruence using either an inches or a dinches scale.

We've seen that the standard representation theorem associated with betweenness and congruence maps pairs of points onto numbers in the ways constrained by $(a)$ and $(b)$ above. But this is only one of many ways we can numerically represent betweenness and congruence. Milne offers an alternative, which replaces $(b)$ above with $(b)$ :

(b) for any points $x, y$, and $z, y$ Bet $x z$ iff $\sqrt{ } d(x, y)+\sqrt{ } d(y, z)=\sqrt{ } d(x, z)$ Note that if we replace $(b)$ with $(b)$, we've effectively constructed a dinches scale (where the length in dinches is the distance between pairs of points). Consider any function $d$ that satisfies $(a)$ and $(b)$; then a function $d^{\prime}$ satisfies $(a)$ and $(b)$ iff $d(x, y)=d^{\prime}(x, y)^{2}$. If $d$ is the inches scale, then $d^{\prime}$ is the dinches scale.

Nothing about the axioms governing betweenness and congruence rules

\footnotetext{
${ }^{14}$ Elsewhere Field writes that the uniqueness theorem "explain[s], in terms of intrinsic facts about space which are statable without such arbitrary choices, why the choice of functions to be invoked in the extrinsic theory will be arbitrary to precisely the extent that it is." (1986, 45-46) (original emphasis)
} 
out ( $b$ ) as opposed to (b). As Milne says, neither (b) nor (b) "represent an intrinsic constraint on distance functions, i.e. an intrinsic feature of length measurement." (Milne 1986, 343) And so we reach the same moral as in section (4): the fundamental distance relations - whether Bet and Cong, or $\succeq$ and $\circ-$ can be mapped onto many different numerical structures. The relations themselves do not place constraints on the numbers we may use to represent them. So the pattern of betweenness and congruence relations simply cannot explain why we use certain numerical representations rather than others. Field's claims to the contrary are mistaken.

While this is all correct, Milne goes on to draw a much more radical conclusion: Field's framework fails to provide an intrinsic explanation of the structure of space. In particular, Milne says, the fact that there are multiple ways of numerically representing the pattern of betweenness and congruence relations entails that the obtaining of some of these relations is a matter of convention. As a result, certain facts about geometry are not "intrinsic facts," and explanations that invoke these facts are not intrinsic explanations.

To illustrate this, Milne introduces the relation of differential congruence. To say that pairs of points $x y$ and $x^{\prime} y^{\prime}$ are differentially congruent to $z w$ and $z^{\prime} w^{\prime}$ means, intuitively, that "the difference between the distance from $x$ to $y$ and the distance from $x^{\prime}$ to $y^{\prime}$ is congruent to the difference between the distance from $z$ to $w$ and the distance from $z^{\prime}$ to $w^{\prime}$." (Milne 1986, 343) Milne then claims that the dinches scale does not preserve the differential congruence relation:

"If one obtains in inches the difference in length between, say, two rigid rods by measuring their lengths then subtracting the smaller from the greater and finds it congruent to the difference between another pair usually the result disagrees with that obtained when the lengths are measured in dinches... Differential congruence is not independent of the measuring procedure by which it is ascertained... What is of the utmost importance here is that differential congruence is not fixed by the intrinsic facts, the facts about betweenness and congruence." (Milne 1986, 343-344)

But Milne's conclusion at the end of the paragraph is not warranted. To see this, note that differential congruence may be defined in terms of congruence:

points $x y$ and $x^{\prime} y^{\prime}$ are differentially congruent to points $z w$ and $z^{\prime} w^{\prime}$ iff for some points $u$ and $v, x^{\prime} u$ Cong $x y, z^{\prime} v$ Cong $z w$, and $u y^{\prime}$ Cong $v w^{\prime}$

Given this, facts about differential congruence must be fixed by the facts about congruence. 
It is easier to see exactly where Milne's argument goes awry if we use the notion of concatenation, which is interdefinable with differential congruence: ${ }^{15}$

points $x y$ and $x^{\prime} y^{\prime}$ are differentially congruent to points $z w$ and $z^{\prime} w^{\prime}$ iff for any objects $l_{1}, l_{2}, l_{3}, l_{4}$, whose endpoints are $x y, x^{\prime} y^{\prime}, z w, z^{\prime} w^{\prime}$, respectively, and some object $o, \circ\left(o, l_{1}, l_{2}\right)$ and $\circ\left(o, l_{3}, l_{4}\right)$

Now consider the following four rods:

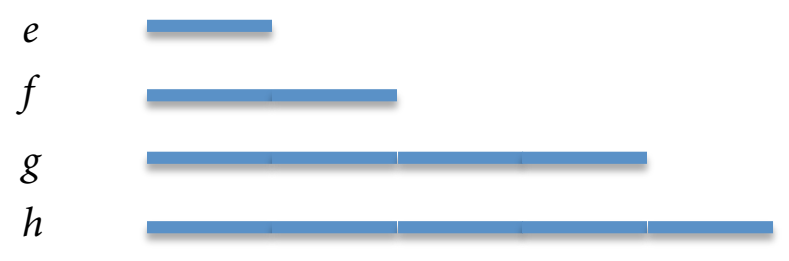

Suppose that $e$ is one inch long (one dinch long), $f$ is two inches long (four dinches long), $g$ is four inches long (sixteen dinches long), and $h$ is five inches long ( 25 dinches long). The difference in the lengths of $e$ and $f$ is one inch, and the difference in the lengths of $g$ and $h$ is one inch. Since one inch $=$ one dinch, the difference in the lengths of $e$ and $f$ is one dinch long, and likewise for the difference in the lengths of $g$ and $h$.

Milne writes that "condition $(b)$ corresponds to the convention that differences in length are treated as distances themselves and their congruence ascertained directly, e.g., one can place rods side by side and measure the overlap of the longer over the shorter. Such a procedure fails for dinches: the directly measured difference in lengths between two rods (in dinches) is not equal to the absolute difference in length of the two rods (again measured in dinches)." (Milne $1986,343)$ The "directly measured difference" in lengths between rods $x$ and $y$ is, intuitively, the length value one would obtain by lining up the rods and using a ruler, say, to measure the length of the part of the longer rod that does not overlap the shorter one. In contrast, the "absolute difference" in length between rods $x$ and $y$ is the length value one would obtain by calculating $|d(x)-d(y)|$, where $d$ is a distance function from rods to numbers.

If $d$ maps rods onto the dinches scale, the "absolute difference" in length between rods $e$ and $f$ is $|4-1|=3$, and the "absolute difference" in length

\footnotetext{
${ }^{15}$ This interdefinability claim works only if we make some ontological assumptions. For instance, we must assume that every object has endpoints, and that for every pair of points $(x, y)$, there is an object that has $x$ and $y$ as endpoints (this object might be the fusion of points between $x$ and $y$ ). Since these ontological issues are irrelevant to the issues I'm concerned with, I put these complications aside.
} 
between rods $g$ and $h$ is $|25-16|=9$. In each case, the "absolute difference" in length between the rods differs from the "directly measured difference" in length between the rods - which in both cases is 1 dinch. If, on the other hand, $d$ maps rods onto the inches scale, the "absolute difference" in length between rods $e$ and $f$ is $|2-1|=1$, and the "absolute difference" in length between rods $g$ and $h \mid 5$ $4 \mid=1$ - both of which equal the "directly measured difference" in length, which is also 1 inch. So, whether the "directly measured difference" value is the same as the "absolute difference" value depends on the scale we use to numerically represent the lengths of these objects.

Milne takes this to show that differential congruence is somehow conventional. But it shows no such thing. The fact that the number assigned to the "directly measured difference" and the number assigned to the "absolute difference" varies with choice of measurement scale does not show that the underlying length facts vary with choice of measurement scale as well.

So what does this show? It shows that the "absolute difference" in length yields the same value as the "directly measured difference" in length only when the measurement scale $d$ is additive. To see this, let's go through two examples. Consider the inches scale, $d_{i n}$. The "directly measured difference" in inches between $l_{1}$ and $l_{2}$ is the value the inches scale assigns to $x\left(d_{i n}(x)\right)$. The "absolute difference" in inches between $l_{1}$ and $l_{2}$ is the value we get when we subtract the value the inches scale assigns to $l_{1}$ from the value it assigns to $l_{2}\left(d_{i n}\left(l_{2}\right)-d_{i n}\left(l_{1}\right)\right)$. As we've seen, the inches scale $d_{i n}$ is additive over the concatenation relation: $\circ(x$, $\left.l_{1}, l_{2}\right)$ iff $d_{i n}(x)+d_{i n}\left(l_{1}\right)=d_{i n}\left(l_{2}\right)$. It follows from this that $d_{i n}(x)=d_{i n}\left(l_{2}\right)-d_{i n}\left(l_{1}\right)$, and thus that the "directly measured difference" in inches $\left(d_{i n}(x)\right)$ will always equal the "absolute difference" in inches $\left(d_{\text {in }}\left(l_{2}\right)-d_{\text {in }}\left(l_{1}\right)\right)$.

Now consider the dinches scale, $d_{d i n}$. The "directly measured difference" in dinches between $l_{1}$ and $l_{2}$ is the value the dinches scale assigns to $x\left(d_{\text {din }}(x)\right)$. The "absolute difference" in dinches between $l_{1}$ and $l_{2}$ is the value we get when we subtract the value the dinches scale assigns to $l_{1}$ from the value it assigns to $l_{2}$ $\left(d_{d i n}\left(l_{2}\right)-d_{d i n}\left(l_{1}\right)\right)$. As we saw in section (4), the dinches scale is not additive over the concatenation relation. Instead, the dinches scale is such that $\circ\left(x, l_{1}, l_{2}\right)$ iff $\left(\sqrt{ } d_{d i n}(x)+\sqrt{ } d_{d i n}\left(l_{1}\right)\right)^{2}=d_{d i n}\left(l_{2}\right)$. It is obvious that, in general, $d_{d i n}\left(l_{2}\right)-d_{d i n}\left(l_{1}\right)$ does not equal $d_{d i n}(x)$. The fact that the dinches scale is not additive over the concatenation relation entails that the "directly measured difference" in dinches will not generally equal the "absolute difference" in dinches.

So Milne is correct to say that the "absolute difference" in dinches does not equal the "directly measured difference" in dinches. But all this means is that the dinches scale is not additive over the standard concatenation relation. And, as we 
have seen, the fact that one can construct a scale of measurement that is not additive over the relevant concatenation relation does not entail anything at all about the fundamental length predicates; and in particular, it does not entail that the "differential congruence is not fixed by the intrinsic facts, the facts about betweenness and congruence." Differential congruence is fixed by the intrinsic facts. What is not fixed by the intrinsic facts is the numerical structure we use to represent those facts. So far, we've seen no reason to think that Field's framework does not provide an intrinsic explanation of the structure of Euclidean geometry.

\section{Milne's Second Objection: Congruence}

Field extends Hilbert's strategy for axiomatizing Euclidean geometry to scalar magnitudes like mass, temperature, and so on. For scalar magnitudes, the basic idea is to introduce different families of betweenness and congruence predicates: intuitively, mass-betweenness and mass-congruence predicates hold among objects with mass, temp-betweenness and temp-congruence predicates hold among objects with temperature, etc. ${ }^{16}$

The axiom system for these scalar notions of betweenness and congruence that Field adopts is in essence the axiom system for an "absolute difference structure" described by Krantz et al. (1970, 171-173) (see also Field 1980, 58 and 119-120)). ${ }^{17}$ And the representation theorem for scalar betweenness and congruence is slightly different from the one used in Euclidean geometry, as it assigns numerical values to individual objects rather than pairs of points:

$<A$, Bet, Cong $>$ is a model of the axioms iff there is a function $f$ from $A$ into the real numbers, such that:

(a) for any $x, y, z$, and $w, x y$ Cong $z w$ iff $|f(x)-f(y)|=|f(z)-f(w)|$

(b) for any $x, y$, and $z, y$ Bet $x z$ iff $f(x) \leq f(y) \leq f(z)$ or $f(z) \leq f(y) \leq f(x)$

The corresponding uniqueness theorem for scalar betweenness and congruence says that, given a model of the axiom system and any two functions $f$ and $f^{\prime}$ defined over $A$, if $f$ satisfies $(a)$ and $(b)$, then $f^{\prime}$ satisfies $(a)$ and $(b)$ iff $f^{\prime}$ is a linear

\footnotetext{
${ }^{16}$ Note that for quantitative features that require an ordering - such as length, mass, temperature, etc. - the predicate less than or equal to (Less) is used in place of betweenness as a primitive notion. (Betweenness can then be defined: $y$ Bet $x z$ iff $x$ Less $y$ Less $z$ or $z$ Less $y$ Less $x$.) For ease of exposition, I follow Milne and ignore this complication.

${ }^{17}$ Krantz et al. employ one primitive: $\succeq$. To say that $x y \succeq z w$ means, intuitively, that the distance between $x$ and $y$ is greater than or equal to the distance between $z$ and $w$. As Field notes, the system Krantz et al. describe can be modified so as to employ Field's primitives instead. One way to do that is to define Bet and Cong in terms of $\succeq: x y$ Cong $z w$ iff $x y \succeq z w$ and $z w \succeq x y$; $x$ Bet $y z$ iff $y z \succeq x y, x z$.
} 
transformation of $f^{18}$

Milne's second objection to Field targets the scalar congruence predicate. He argues that whether $x y$ Cong $z w$ obtains depends on the scale of measurement we use; and since choice of measurement scale is a matter of convention, then so is the obtaining of the congruence predicate. Therefore, any fact involving congruence is not "an intrinsic fact," and thus congruence is "not intrinsic."(Milne 1986, 344) ${ }^{19,} 20$

(It is interesting to note that while Milne challenges the intrinsicality of congruence, he does not doubt the intrinsicality of betweenness. ${ }^{21}$ It is unclear why congruence is a target of suspicion while betweenness is not. If the fact that there are multiple different relations among numbers can be used to represent facts about congruence shows that these facts about congruence are not intrinsic, as Milne believes, then the same can be said for betweenness. ${ }^{22}$ )

Milne's argument goes as follows. One can measure the same scalar using different measurement scales, where these measurement scales are not linear transformations of one another. Such scales differ not only on the values they assign to the same interval, but on whether congruent intervals are assigned the same value. One example Milne offers is the Dalton temperature scale, which is "logarithmically related to the absolute scale." Suppose that $a, b, c$, and $d$ are objects with temperature, and suppose that $a b$ Temp-Cong $c d$. The absolute scale assigns the same number to $a b$ and $c d$, while the Dalton scale does not. From this fact, Milne draws the following conclusion: "The moral is that [scalar]-congruence is not independent of the manner in which it is ascertained. And so, it seems, it is not the case that [scalar]-congruence is intrinsic." (Milne 1986, 345)

But this argument is not valid. The fact that intervals assigned the same value on the absolute scale are assigned different values on the Dalton scale does not entail anything about the intrinsicality of congruence. All it shows is that a relational structure $<A$, Temp-Bet, Temp-Cong $>$ can be mapped to many

\footnotetext{
${ }^{18} \varphi$ is a linear transformation of $\phi$ iff $\varphi=a \phi+b$, where $a$ and $b$ are real numbers (and $a \neq 0$ ).

${ }^{19}$ Recall that, for Milne, $x y$ Cong $z w$ is intrinsic iff $x y$ Cong $z w$ obtains in virtue of the objects and the relations that hold among them, and nothing else.

${ }^{20}$ This line of thought is similar to Ellis's, who likewise appeared to believe that if choice of measurement scale is a matter of convention, then so is the fundamental measuring procedure.

21 "It is abundantly clear from Field's discussion that he regards $\phi$-betweenness and $\phi$-congruence as relations whose obtaining is an intrinsic fact. About $\phi$-betweenness there is no ground for complaint. $\phi$-congruence is quite another matter." (Milne 1986, 344)

${ }^{22}$ A similar point applies to Ellis: Ellis doubts the objectivity of concatenation, but not of ordering $(\succeq)$. But again, if the fact that there are multiple different relations among numbers that can be used to represent facts about concatenation shows that these facts about concatenation are not objective, as Ellis believes, then the same can be said for ordering.
} 
different numerical structures. Some will represent congruent intervals with the same number, others will not. Some are more familiar and convenient in some contexts, while others are more familiar and convenient in other contexts. But the fact that the choice of numerical structure is a matter of convention does not entail that the obtaining of the underlying temperature relations is not intrinsic. ${ }^{23}$

In sum, Milne is correct in pointing out that the choice of numerical structure is not fixed by the pattern of betweenness and congruence relations, and thus that the pattern of betweenness and congruence relations cannot provide an intrinsic explanation of our standard numerical representations of length, mass, temperature, and so on. But this does not show that the underlying betweenness and congruence relations are in any way extrinsic, conventional, or non-objective. Any argument for this conclusion - that the underlying betweenness and congruence relations fail to provide intrinsic explanations for the structure of space and the structure of scalar magnitudes - that relies on premises concerning our representation of this structure is invalid.

\section{A Suggestion}

There seems to be a kind of tension prompting much of the preceding discussion of measurement scales. On the one hand, certain kinds of measurement scales seem, at least at first, to do a worse job of representing reality than others. For instance, the dinches scale seems, intuitively, to represent the underlying length relations in a particularly opaque way, while the inches scale does so in a particularly transparent way. Similarly, the Dalton temperature scale, while surely useful in many contexts, nonetheless does not seem to represent the underlying temperature relations as perspicuously as the absolute temperature scale (there is a reason only one of these scales is called the "absolute" scale).

On the other hand, there does not seem to be any way to justify the intuition that some scales fail to represent the world in as transparent a manner as other scales. The underlying relations of distance, length, mass, temperature, and so on, can be represented by many different numerical structures. Consider the axiomatization of extensive measurement, from Krantz et al., that employs the basic predicates $\succeq$ and $\circ$. Why should $\succeq$ be carried to the numerical relation $\geq$, and why should $\circ$ be carried to the numerical relation + ? Or consider the congruence and betweenness predicates of Field's framework. Why should Temp-Cong, say, be carried to the numerical relation given by $(a)$, and why should Temp-Bet be carried to the numerical relation given by $(b)$ ?

\footnotetext{
${ }^{23}$ See also Krantz et al. (1971, 152).
} 
If there were some way to justify the intuition that some scales do a better job at representing reality than others, then we would have grounds for rejecting odd or unnatural scales like the dinches scale. Even better would be some way to vindicate Field's claim that the underlying relations themselves provide an intrinsic explanation that justifies the use of some scales over others.

Let me here suggest a route that one might take. The standard representation theorem for extensive measurement carries $\succeq$ to $\geq$, and $\circ$ to + . One way to argue that this representation is better than others is to claim that $\succeq$ stands in a privileged relationship with $\geq$, and $\circ$ stands in a privileged relationship with + . But what is this privileged relationship?

One could claim that this relationship is sui generis, but that would not be particularly explanatory. A more interesting option is to claim that this relationship is identity: $\succeq$ is identical to $\geq$, and $\circ$ is identical to + . In other words, the greater than or equal to and addition relations that numbers stand in simply are the greater than or equal to and concatenation relations invoked in extensive measurement. Similarly, one might say, the betweenness and congruence relations among numbers (which we rather clumsily analyze in terms of $\leq$, subtraction, and absolute value) simply are the betweenness and congruence relations invoked in Field's framework. If that is so, then it seems we do have an intrinsic explanation for why we use some scales rather than others: the perspicuous scales are the ones where the numbers representing the scalar magnitudes stand in the very same relations as the scalar magnitudes themselves.

This suggestion, however, requires some revision of Field's framework. Field's primary aim is to provide a nominalistic treatment of spacetime and of quantity - an account of these that does not quantify over properties or relations. In order to accommodate various families of quantities, Field introduces a different betweenness and congruence predicate for each family: Mass-Bet is distinct from Temp-Bet, Mass-Cong is distinct from Temp-Cong, and so forth. But if there are multiple distinct betweenness and congruence predicates, then we cannot implement the suggested strategy. Given Field's framework, Mass-Bet and Temp-Bet are not identical. But, by transitivity of identity, if Mass-Bet is identical to the betweenness relation among numbers, and Temp-Bet is identical to the betweenness relation among numbers, then Mass-Bet must be identical to TempBet.

So in order to implement this strategy, we must accommodate the fact that there are various families of quantities, without having different betweenness and congruence predicates for each family of quantities. To do this, we introduce determinate properties for every family: one gram mass, two grams mass, etc., one 
degree Fahrenheit, two degrees Fahrenheit, etc., and so on. These properties stand in second-order betweenness and congruence relations: two grams mass is between one gram mass and three grams mass, and so a perspicuous mass scale is one where the number used to represent two grams mass is between the numbers used to represent one gram mass and three grams mass. ${ }^{24}$ Likewise for temperature, charge, lengths, and all other scalar magnitudes. On this proposal, then, we do not have to introduce different betweenness and congruence relations for each family of quantities. Since there is only one betweenness relation, and only one congruence relation, we can identify these relations with the corresponding relations that hold among numbers.

If we adopt this proposal, we must quantify over first-order properties, and so we must give up on Field's nominalism. Whether one is amenable to this move depends on one's other metaphysical commitments. I've argued elsewhere that there are independent reasons to be a realist about determinate quantitative properties, and to take the relations that these properties stand in as part of the fundamental structure of the world. ${ }^{25}$ To the extent to which one feels that scales like the dinches scale do not latch onto reality in quite the right way, whereas the scales like the inches scale do, one might take this to be yet another reason to reject the nominalist position. ${ }^{26}$

\footnotetext{
${ }^{24}$ Mundy (1987) offers an account of quantity that posits first-order determinate properties that stand in second-order relations. Rather than use second-order betweenness and congruence relations, he uses second-order less than or equal to and concatenation relations, modeling his account on Krantz et al.'s treatment of extensive measurement.

${ }^{25}$ Eddon (2013)

${ }^{26}$ Many thanks to Chris Meacham and Katia Vavova for comments and discussion.
} 


\section{References}

Eddon, M. (2013). “Fundamental Properties of Fundamental Properties.” Oxford Studies in Metaphysics, Vol. 8. Oxford.

Ellis, Brian (1960). "Some Fundamental Problems of Direct Measurement." Australasian Journal of Philosophy 38: 37-47.

Ellis, Brian (1966). Basic Concepts of Measurement. Cambridge University Press.

Field, Hartry (1980). Science Without Numbers. Princeton University Press.

Fox, John (2007). “Why We Shouldn't Give Ellis a Dinch.” Analysis 67: 301-303.

Krantz, D., Luce, R., Suppes, P, and Tversky, A. (1971). Foundations of Measurement: Volume I. Dover.

Lewis, David (1983). "New Work for a Theory of Universals.” Australasian Journal of Philosophy 61: 343-377.

Milne, Peter (1986). "Hartry Field on Measurement and Intrinsic Explanation." British Journal for the Philosophy of Science 37: 340-346.

Mundy, Brent (1987). “The Metaphysics of Quantity.” Philosophical Studies 51: 29-54. 